\title{
Entropy and scintillation analysis of acoustical beam propagation through ocean internal waves
}

\author{
Andrey K. Morozov and John A. Colosia) \\ Woods Hole Oceanographic Institution, Woods Hole, Massachusetts 02543
}

(Received 9 January 2004; revised 5 August 2004; accepted 5 August 2004)

\begin{abstract}
Parabolic equation numerical simulations of waveguide acoustical beam propagation in an ocean of Garrett-Munk internal waves are used to examine the range evolution of beam properties such as beamwidth (both spectral and spatial), Shannon entropy, and scintillation index, as a function of beam angle. Simulations are carried out at $250-$ and $125-\mathrm{Hz}$ acoustic frequencies. The ray trajectories associated with these beams are predominantly chaotic or exponentially sensitive to initial conditions and/or medium perturbations. At long range near saturation, the finite-frequency beams show a constant rate of change of Shannon entropy with range, independent of acoustic frequency. This full-wave rate of entropy is of the same order of magnitude as the average rate of entropy for the ray trajectories associated with this beam. Finite-range Lyapunov exponents provide the estimates of ray entropy rate or Kolmogorov-Siani entropy. The correspondence between full-wave and ray entropies suggests a full-wave manifestation of ray chaos, but only once statistical saturation is obtained. In spite of this correspondence, the simulated acoustical beams expand diffusively not exponentially (or explosively). (C) 2005 Acoustical Society of America.
\end{abstract}

[DOI: $10.1121 / 1.1854571]$

PACS numbers: $43.60 . \mathrm{Cg}$, 43.30.Ft, 43.30.Cq [AIT]

Pages: $1611-1623$

\section{INTRODUCTION}

Scattering by small-scale ocean processes like internal waves imposes the ultimate limitations on large-scale ocean acoustic remote sensing and matched-field processing. Further, the stochastic properties of the sound field scattered by internal waves are poorly understood in long-range propagation as conditions of saturation are established (Colosi et al., 1999a,b). Recent progress in utilizing ray methods to understand scattering processes in long-range acoustic propagation suggests there is an exponential sensitivity to initial conditions and a rapid growth of acoustic field complexity with a scale of a few hundred kilometers (Brown et al., 2003; Beron-Vera et al., 2003). However, as is well known in other fields of wave propagation, finite-frequency effects can slow, suppress, or mask this increasing complexity (Casati, 1996). The objective of this paper is to examine the phenomenon of ocean acoustic ray chaos and its potential manifestations in finite-frequency wave fields, by the method of numerical simulation. This same question has received much attention in the quantum chaos field (Gianonni et al., 1989; Casati and Chirikov, 1995) and is often termed wave chaos.

From an ocean acoustic perspective and motivated by the work of Wolfson and Tappert (2000), this problem was initially considered by Tappert (2003), who hypothesized that the width of a narrow-angle beam propagating through a strong mesoscale sound-speed field would increase exponentially (explosively) with range at a rate that is predicted by the geometric Lyapunov exponent. Tappert (2003) examined this problem in detail for the case of no waveguide and a single-scale random medium where he showed theoretically

\footnotetext{
a) Author to whom correspondence should be addressed. Electronic mail: jcolosi@whoi.edu
}

the possibility of observing explosive beam growth between the limits of short-range dominance by diffraction and longrange dominance of uncorrelated multipath (or saturation). Indeed, some recent results for an equivalent problem involving a $2 \mathrm{D}$ electron gas have demonstrated this exponential growth (Topinka and Westervelt, 2003).

Inspired by Tapperts' work, this problem of ocean acoustic wave chaos is examined in the presence of a waveguide and realistic ocean sound-speed fluctuations for which the problem of finding a full-wave manifestation of ray chaos can be different. Using parabolic equation (PE) and ray theory numerical simulations in the presence of ocean soundspeed fluctuations obeying the Garrett-Munk (GM) internal wave model, PE beam simulations are done to mimic a small-angle bundle of rays. To test the frequency dependence of the results, simulations at the two frequencies of 125 and $250 \mathrm{~Hz}$ are done. An example of unperturbed and perturbed $250-\mathrm{Hz}$ PE beams for an axial angle of about $5 \mathrm{deg}$ is shown in Fig. 1 (bottom panels). Unlike the previous analysis (Tappert, 2003), more acoustic observables than simply beamwidth are considered. Importantly, we consider the Shannon entropy of vertical profiles of the beam complex envelope as a function of range, $E(r)$, because of the close connection between the instability of the ray equations (i.e., Lyapunov exponent) and the Kolmogorov-Sinai (KS) entropy (actually a rate). Recent results have shown that the rate of change of Shannon entropy, $E(t)$, for some idealized dynamical systems is closely related to the KS entropy over an intermediate regime of times (Latora and Baranger, 1999). ${ }^{1}$ Thus, the rate of Shannon entropy for our finite-frequency numerical simulations can be directly compared to ray simulation results of average finite-range Lyapunov exponent (a measure of KS entropy).

In this study, it is found that acoustical beams in an 


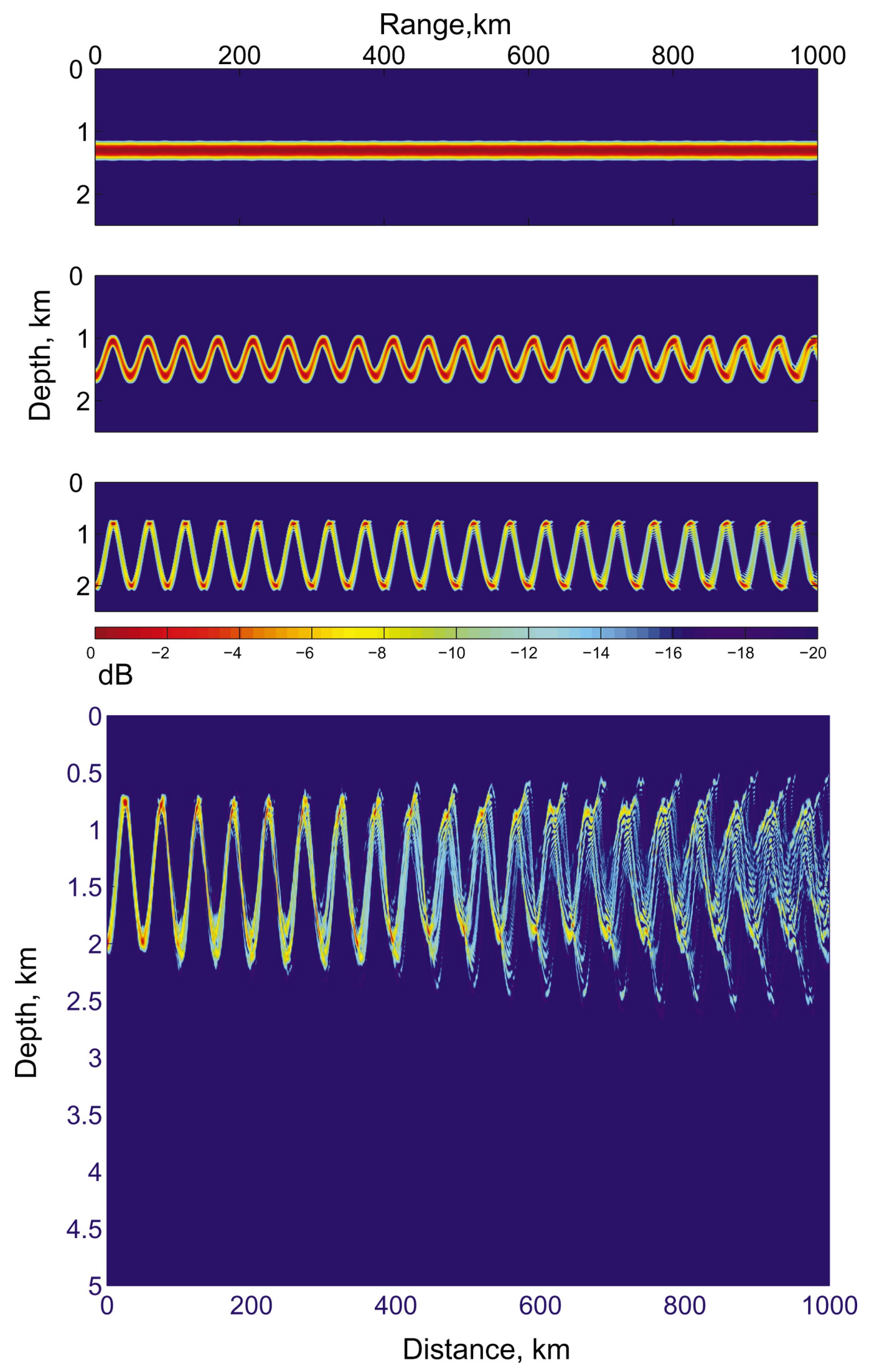

FIG. 1. Examples of parabolic equation calculations of four different weakly divergent acoustical beams. In the upper three panels the simulations were done without internal wave sound-speed perturbations and the source was placed at depths of $1300 \mathrm{~m}$ (axial depth; upper), $1600 \mathrm{~m}$ (second upper), and $2000 \mathrm{~m}$ (third upper). In the lowest panel is an example of the 2000-m source depth acoustical beam with internal-wave sound-speed perturbations. These simulations were carried out at $250 \mathrm{~Hz}$ using the Munk canonical profile. Intensity is in $\mathrm{dB}$ referenced to the maximum intensity. 
ocean waveguide with realistic sound-speed perturbations from internal waves do not expand exponentially (or explosively), but they expand diffusively (Fig. 1). Moreover, it is also found that as the beam fills the waveguide and the wave function behaves like Gaussian random noise (i.e., full saturation), $E(r)$ varies linearly with range. The rate of Shannon entropy in this linear regime is computed to be between 0.06 and $0.11 \mathrm{bit} / \mathrm{km}$ independent of frequency, and the smaller values are associated with the higher angle beam. ${ }^{2}$ These values are to be compared to at least twice the KS entropy (which lacks phase information) and are computed to be between 0.02 and $0.04 \mathrm{bit} / \mathrm{km}$, with again smaller entropy rates associated with higher angle rays. Clearly, the wave field entropy cannot grow indefinitely as this system is bounded by the ocean surface and bottom. We surmise that the entropy will grow linearly until acoustic energy fills the entire water column, at which time the entropy should stabilize. The diffusive beam spreading and the linear rate of Shannon entropy are the same in both the $125-$ and $250-\mathrm{Hz}$ simulations. These results suggest a finite-frequency manifestation of ray chaos, but only after the establishment of saturation, where the scintillation index is close to 1 . Thus, Tappert's conjecture that there should be a full-wave manifestation of ray chaos is confirmed but in a different way than anticipated from the homogeneous background case. That is, in the case of a waveguide, the full-wave manifestation of ray chaos is seen in the entropy, not in the beam spread.

The organization of the paper is as follows. In Sec. II the acoustic propagation and internal wave models which are the basis of the Monte Carlo numerical simulation method are discussed. The narrow angle beam is described in Sec. III. In Secs. IV and V the range evolution of beam intensity statistics and beamwidth are described. Section VI presents results on beam entropy, while Sec. VII presents results on the connection between wave and ray entropies. Summary and conclusions are given in Sec. VIII.

\section{SOUND PROPAGATION SIMULATION}

The sound speed as a function of range $(r)$, and depth $(z)$ is defined by the sum

$$
c(r, z)=c_{m}(z)+\delta c(r, z),
$$

where $c_{m}(z)$ is the mean sound-speed profile and $\delta c(r, z)$ is a random perturbation induced by internal waves. For simplicity, a canonical model is used for the mean profile (Munk, 1976) and, the internal wave contribution is modeled using the Garrett-Munk spectrum which has been described in detail by Colosi and Brown (1998). For the buoyancy frequency, $N(z)$, a canonical form is also used such that, $N$ $=6 \exp (z / 1300) \mathrm{cph}$. For the internal wave simulations 50 vertical modes and $2^{12}$ horizontal wave numbers in each of the horizontal directions are used, with a horizontal step of $25 \mathrm{~m}$. The GM spectrum is cut off at a maximum horizontal wave number of $4.0 \mathrm{cpkm}$, and the internal wave energy was normalized so that at the depth where $N=3 \mathrm{cph}$ the rms internal wave displacement is equal to $7.3 \mathrm{~m}$. The potential gradient of sound speed is proportional to $N^{2}(z)$ as in Colosi and Brown (1998). Two hundred realizations of the random sound-speed fields were calculated and used to compute 200 realizations of the acoustic pressure field for the various beam geometries out to $2000-\mathrm{km}$ range. The acoustic frequencies were 125- and $250 \mathrm{~Hz}$. The sound-pressure propagation modeling was carried out using a wide-angle parabolic equation (PE) approach (Jensen et al., 2000). The PE solution provides the complex envelope $\psi(r, z)$, which is assumed to vary slowly in range. The full-wave field can be written as

$$
p(r, z)=\psi(r, z) H_{0}^{1}\left(k_{0} r\right),
$$

where $H_{0}^{1}$ is the Hankel function, which satisfies the wellknown Bessel equation. The Hankel function can be replaced by its asymptotic form for $k_{0} r \gg 1$, giving

$$
H_{0}^{1}\left(k_{0} r\right)=\left(\frac{2}{\pi k_{0} r}\right)^{1 / 2} \exp \left(i\left(k_{0} r-\pi / 4\right)\right) .
$$

In the subsequent analysis all cylindrical spreading factors were removed. In this paper a numerical implementation of the PE solution is used which is based on the Greene approximation as described in Jensen et al. (2000). For the starting field an analytical Gaussian source function was used of the form

$$
\psi(0, z)=\left(\frac{2 k_{0}^{2}}{\pi \Delta^{2}}\right)^{1 / 4} \exp \left(-k_{0}^{2}\left(z-z_{s}\right)^{2} / \Delta^{2}\right),
$$

where $z_{s}$ is the source depth, and the width factor $\Delta$ was adjusted to values between 60 and 100 to achieve weakly diverging beams, as will be discussed in the next section.

Before we leave this subject, it must be noted that a few simulations were carried out using the standard parabolic equation (SPE) for the envelope which has the form

$$
\begin{aligned}
\frac{i}{k_{0}} \frac{\partial \psi}{\partial r} & =-\frac{1}{2 k_{0}^{2}} \frac{\partial^{2} \psi}{\partial z^{2}}-U(r, z) \psi=0, \\
U(r, z) & =\frac{1}{2}\left[\left(\frac{c_{0}}{c(r, z)}\right)^{2}-1\right] \simeq-\left[\frac{c_{m}-c_{0}}{c_{0}}+\frac{\delta c}{c_{0}}\right],
\end{aligned}
$$

where $k_{0}=\omega / c_{0}$ is a reference wave number. It was found that the statistics of the beams was insensitive to whether the SPE or the larger-angle PE were used in the computation.

\section{WEAKLY DIVERGENT BEAMS}

The existence of weakly divergent bundles of rays was discovered and described by Brekhovskih et al. (1995), Goncharov and Kurtepov (1994), and Petukhov (1994). Longrange propagation experiments often showed strong increases of sound intensity at different depths far from the axis of the underwater channel. This phenomenon was explained by focusing of acoustical energy along weakly divergent bundles of rays. Rays comprising this bundle are grouped around a central ray with the turning point at the source depth, i.e., the beam emerges horizontally from the source. When a sound source is near the sound-channel axis, 


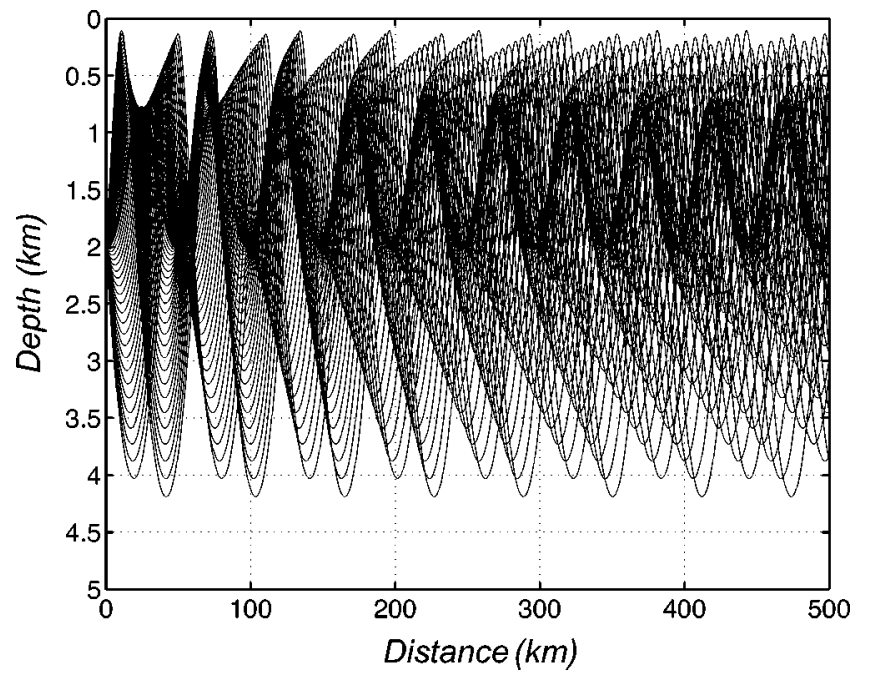

FIG. 2. Concentration of ray trajectories along the weakly divergent bundle of rays. The numerical simulation was performed for a Munk canonical sound-speed profile.

this bundle corresponds to the usual energy concentration along the channel axis. The angles of weakly divergent bundle of rays are close to points of smooth extremum of the angular dependence of a ray cycle length (Brekhovskih et al., 1990). It must be noted, however, that for the adopted canonical profile in this study, the Brekhovskih condition does not apply since the ray cycle distance is a monotonic function of ray grazing angle. Nevertheless, in Fig. 2 one can see how energy and ray trajectories are concentrating near the direction of weakly divergent bundle of rays for this canonical waveguide (Munk, 1976). Such concentration can be found in any horizontally homogeneous ocean profile and this property remains (in the adiabatic approach) for small horizontal gradients of sound speed. A further geometrical acoustics interpretation of a narrow weakly divergent bundle of rays is demonstrated in Fig. 3. Suppose that the source radiation width is confined by crossing trajectories of two acoustic rays shifted in the horizontal direction by a small distance roughly equal to the horizontal width of bundle; then, the sound energy will spread in the narrow path bounded by those rays. A sound source with such directivity will form a narrow weakly divergent beam radiated at zero

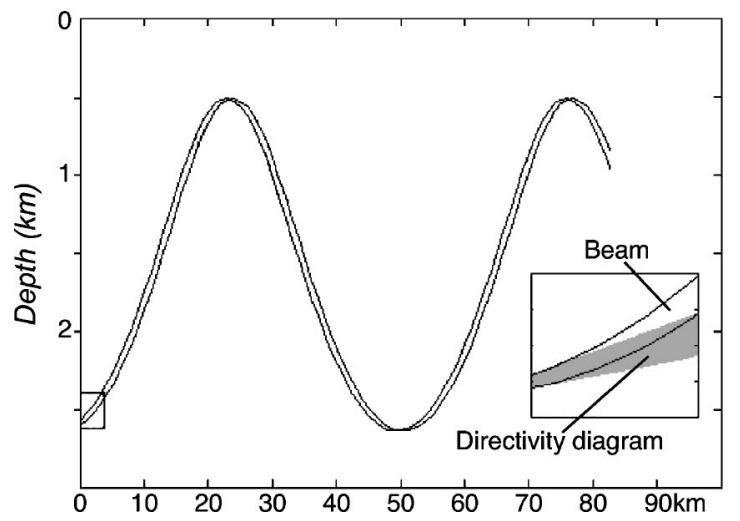

FIG. 3. Ray diagram of two paths slightly displaced in range to demonstrate generation of a weakly divergent beam.

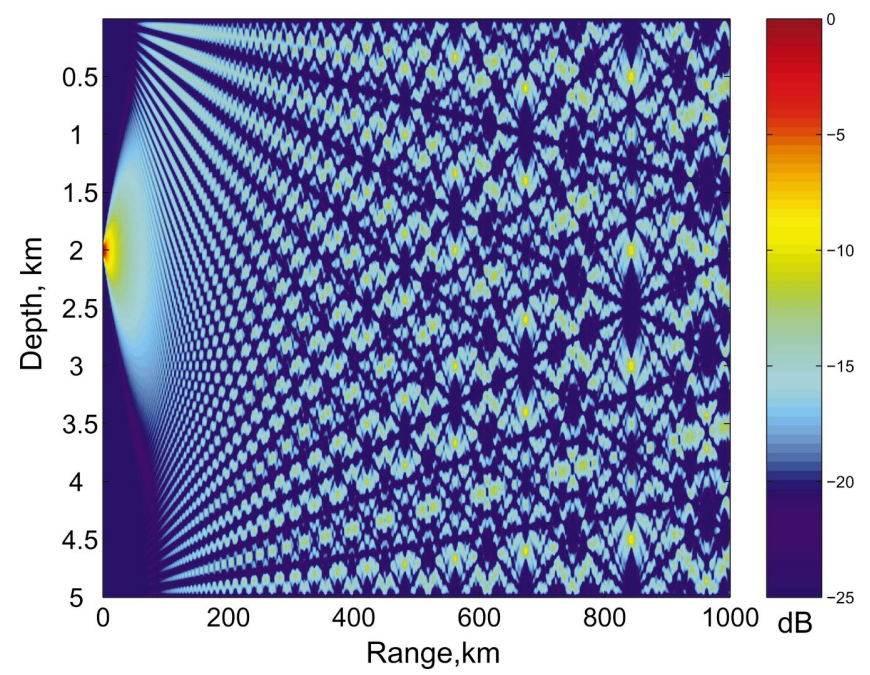

FIG. 4. Sound emitted by the same aperture as the 2000-m source beam in Fig. 1 (lower), but here there is a constant background sound speed.

initial angle. This property can be used to simulate a very narrow-angle beam emitting from a rather small, finite vertical aperture. It can be achieved by variation of the initial Gaussian beamwidth, $\Delta$, until the condition of a weakly divergent beam is satisfied. Each turning point acts like a local focusing lens.

The focusing effect partially compensates the diffractive expansion, thereby forming the weakly divergent beam. An example of a $250-\mathrm{Hz}$ weakly divergent beam is shown in Fig. 1. The vertical dimension of the 2000-m-depth sound source aperture is roughly $150 \mathrm{~m}(\Delta=60)$. The sound from a source with the same aperture as Fig. 1 was numerically simulated in a homogeneous medium, where $H$ is the ocean depth (Fig. 4). A comparison between Figs. 1 and 4 shows that in a waveguide even a relatively small aperture sound source can concentrate energy in a very narrow beam. The same property can be used in receiving arrays for forming super-resolution directivity by taking into account the soundspeed profile $c(z)$ measured near the receiver (Dzieciuch, Munk, and Worcester, 2001).

The question naturally arises: Can these narrow beams be constructed in such a way as to be absolutely nondivergent? The previous example (Fig. 2) shows that a nondivergent beam is possible in the high frequency or ray limit, but the realization of such a beam at finite frequencies is an interesting problem in underwater acoustics. Nevertheless, on a practical note experimentally, it appears that these beams would be rather easy to generate, as the vertical aperture required is relatively small. An acoustic source array with such an aperture could be constructed for high frequencies, while for low frequencies the beam could be generated using a synthetic vertical aperture in which a source is moved vertically during the signal transmission.

The aforementioned concentrated narrow-angle beams are a simple and interesting subject for numerical simulation of acoustic energy scattering by internal waves, because these beams can mimic the propagation of a narrow bundle of rays, while retaining the computational efficiency of a 

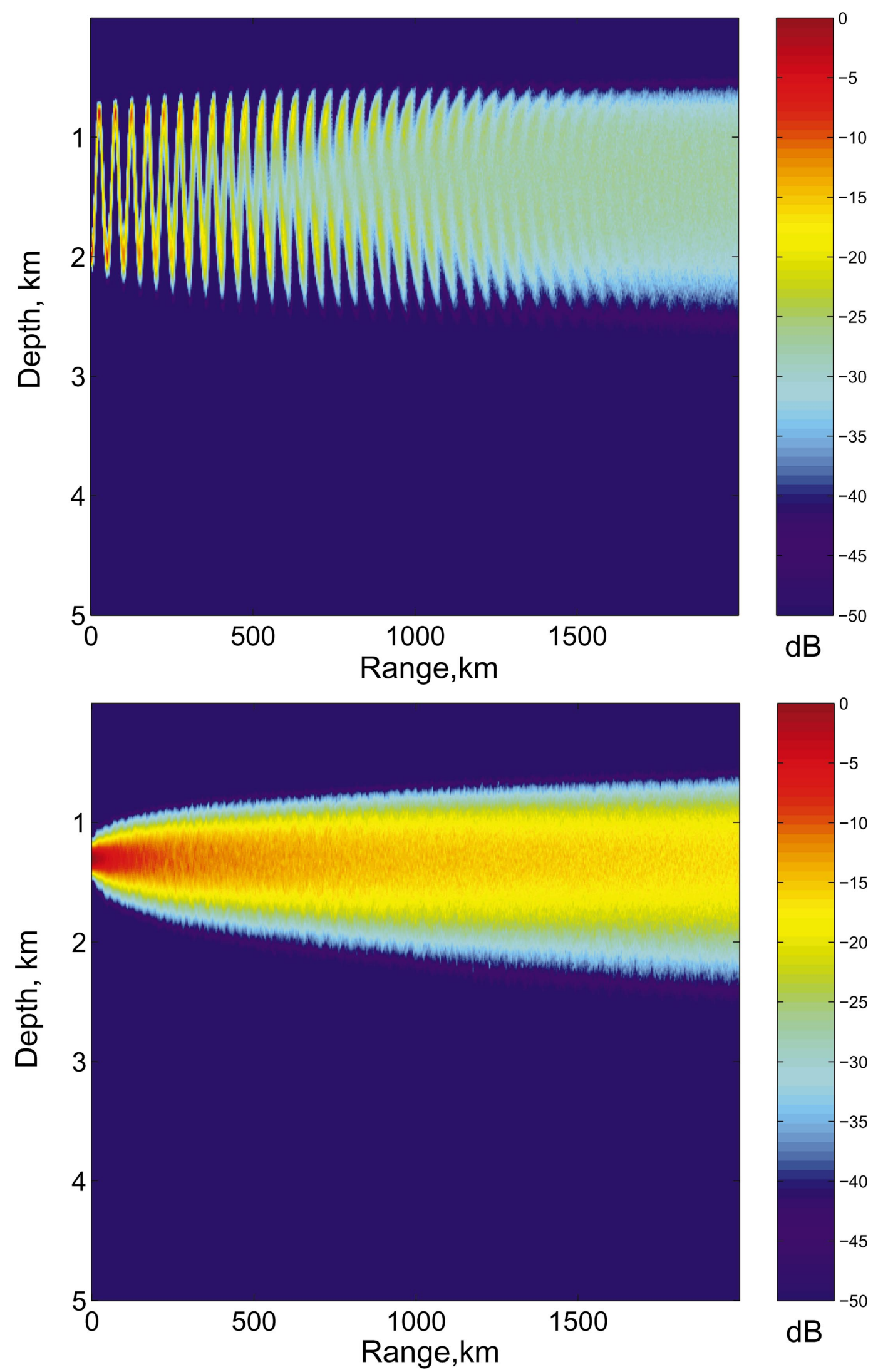

FIG. 5. Range evolution of mean intensity for the $250-\mathrm{Hz}$ beam with source depths of $2000 \mathrm{~m}$ (upper) and $1300 \mathrm{~m}$ (lower).

single-frequency calculation. Clearly, broadband calculations can more narrowly define this bundle of rays by separating multipaths in time, but these calculations are beyond the scope of the present analysis.

\section{INTENSITY FLUCTUATIONS}

In this section intensity fluctuations are analyzed to uncover how the real and imaginary parts of $\psi$ approach uncor- 
related Gaussian random variables or full saturation (Flatté et al., 1979). In full saturation, the scintillation index

$$
\mathrm{SI}=\frac{\left\langle I^{2}\right\rangle}{\langle I\rangle^{2}}-1
$$

approaches the value of 1 (Flatté et al., 1976), and this approach can be from either above or below (Colosi and Baggeroer, 2004). Furthermore, the Gaussian approximation allows a simplified calculation of the Shannon entropy, which will be discussed in Sec. VI. Figure 5 shows the mean intensity, ensemble averaged over 200 internal wave realizations, for a narrow $250-\mathrm{Hz}$ beam with source depths of 1300 and $2000 \mathrm{~m}$. For the 2000-m beam acoustical energy is seen to scatter and diffuse from the narrow path, in-filling the axial depth region with energy and slowly spreading the energy towards the ocean boundaries. The axial beam, of course, only shows the slower outward spreading towards the boundaries. Figure 6 shows the spatial distribution of SI for the same beams. To avoid problems of division by zero, the SI is only calculated for regions of space where there is significant average intensity. At short ranges SI is increasing, while at the longest ranges near saturation values of 1 are observed more-or-less uniformly over the depth of the beam between 750 and $2000 \mathrm{~m}$. In the midranges SI has significant spatial variability, and values that are much larger than 1 . As the beam is breaking up, strong focuses leading to SI values significantly above 1 are observed near the caustics or vertical turning points of the beams, while near the axis the SI values are only slightly higher than 1 . As the vertical caustic structure of the beam disintegrates around 1500-m range, the SI values become more uniform in depth, leading to an approach to saturation from above 1 for the 2000-m beam, but an on-axis approach from below 1 for the 1300-m beam.

A different way of examining the scintillation curves for several source depths simultaneously is to plot the SI values as a function of range, but along a curve defined by the energy centroid of the unperturbed beam. These curves are shown in Fig. 7 for the 250-Hz beams. As previously shown in Fig. 6, the 2000-m source depth beam approaches saturation from above 1, and a similar trajectory is found for a 4000-m source depth. However, for near-axial beams the approach to saturation is quite different. Here, SI approaches 1 from below, and these beams reach saturation at a much earlier range.

The $125-\mathrm{Hz}$ calculation shows very similar behavior as compared to the $250-\mathrm{Hz}$ calculation, except in the following respects. The high-angle beams do not show as large SI values above 1, and the initial growth of SI at short range is slower by about one-half as expected from weak fluctuation theory. For the near-axial beams the same approach to saturation from below 1 is seen, and as in the high-angle case the initial growth of SI is slower by about one-half.

\section{BEAM SPREAD}

Next, the issue of vertical beam spread is addressed, and it proves useful to provide the following definitions for the quantitative description of the acoustical beam evolution. First, the wave function is normalized at each range $r$ so that

$$
\psi_{0}(r, z)=\psi(r, z)\left(\int_{-H}^{0}|\psi(r, z)|^{2} d z\right)^{-1 / 2},
$$

where $H$ is the ocean depth. Thus, the normalized complex envelope has unit power. The central depth $\hat{z}(r)$ and rms width $w(r)$ of a narrow beam at the range $r$ are defined as

$$
\begin{aligned}
& \hat{z}(r)=\int_{-H}^{0} z\left|\psi_{0}(r, z)\right|^{2} d z, \\
& w(r)=2\left(\int_{-H}^{0}(z-\hat{z}(r))^{2}\left|\psi_{0}(r, z)\right|^{2} d z\right)^{1 / 2} .
\end{aligned}
$$

The spatial spectrum of the beam can be defined using the Fourier transformation, so that

$$
S\left(r, k_{z}\right)=\int_{-H}^{0} \psi(r, z) \exp \left(-i k_{z} z\right) d z .
$$

In complete analogy to the spatial domain analysis, in wave number space there is the normalized spatial spectrum $S_{0}$, the center wave number $\hat{k}_{z}$, and the rms spectrum width $w_{s}$, all defined as

$$
\begin{aligned}
& S_{0}\left(r, k_{z}\right)=S\left(r, k_{z}\right)\left(\int_{-\left(k_{z}\right)_{\max }}^{\left(k_{z}\right)_{\max }}\left|S\left(r, k_{z}\right)\right|^{2} d k_{z}\right)^{-1 / 2}, \\
& \hat{k}_{z}(r)=\int_{-\left(k_{z}\right)_{\max }}^{\left(k_{z}\right)_{\max }} k_{z}\left|S_{0}\left(r, k_{z}\right)\right|^{2} d k_{z}, \\
& w_{s}(r)=2\left(\int_{-\left(k_{z}\right)_{\max }}^{\left(k_{z}\right)_{\max }}\left(k_{z}-\hat{k}_{z}(r)\right)^{2}\left|S_{0}\left(r, k_{z}\right)\right|^{2} d k_{z}\right)^{1 / 2} .
\end{aligned}
$$

In this representation the spatial and spectral widths roughly obey an "uncertainty" relation, $w(r) w_{s}(r) \simeq 1$, so that the spatial width, $w(r)$, gives information about the large-scale beam variability, while $w_{s}(r)$ gives information about the high wave number, small-scale beam variability. Figures 5 and 8 show the average spatial and spectral patterns of the 250-Hz beams with source depths at 1300 and $2000 \mathrm{~m}$. These figures show that the $2000-\mathrm{m}$ beams are spreading in such a way as to in-fill the axial region, to make the energy density more-or-less uniform in depth between the unperturbed beam turning points, and to "whiten" the spatial spectrum between the maximum/minimum wave numbers of the unperturbed beam. In addition to the in-filling and spectral whitening, there is a slow expansion of the beam towards the ocean boundaries (spatial) and toward high vertical wave number (spectral), which is the dominant mechanism of change for the 1300-m beam.

Using the spatial and spectra widths of the beams, defined from Eqs. (10) and (14), the range evolution of several $250-\mathrm{Hz}$ beams is shown in Fig. 9. In both cases the short ranges show deterministic variability of the beams over a beam cycle, which gradually damps out to more smooth variability with range as saturation becomes established. In all cases the beamwidth defined either spatially or spectrally increases much more slowly than exponentially. The range variations of $w(r)$ and $w_{s}(r)$ in Fig. 9 is very close to $r^{1 / 2}$, 

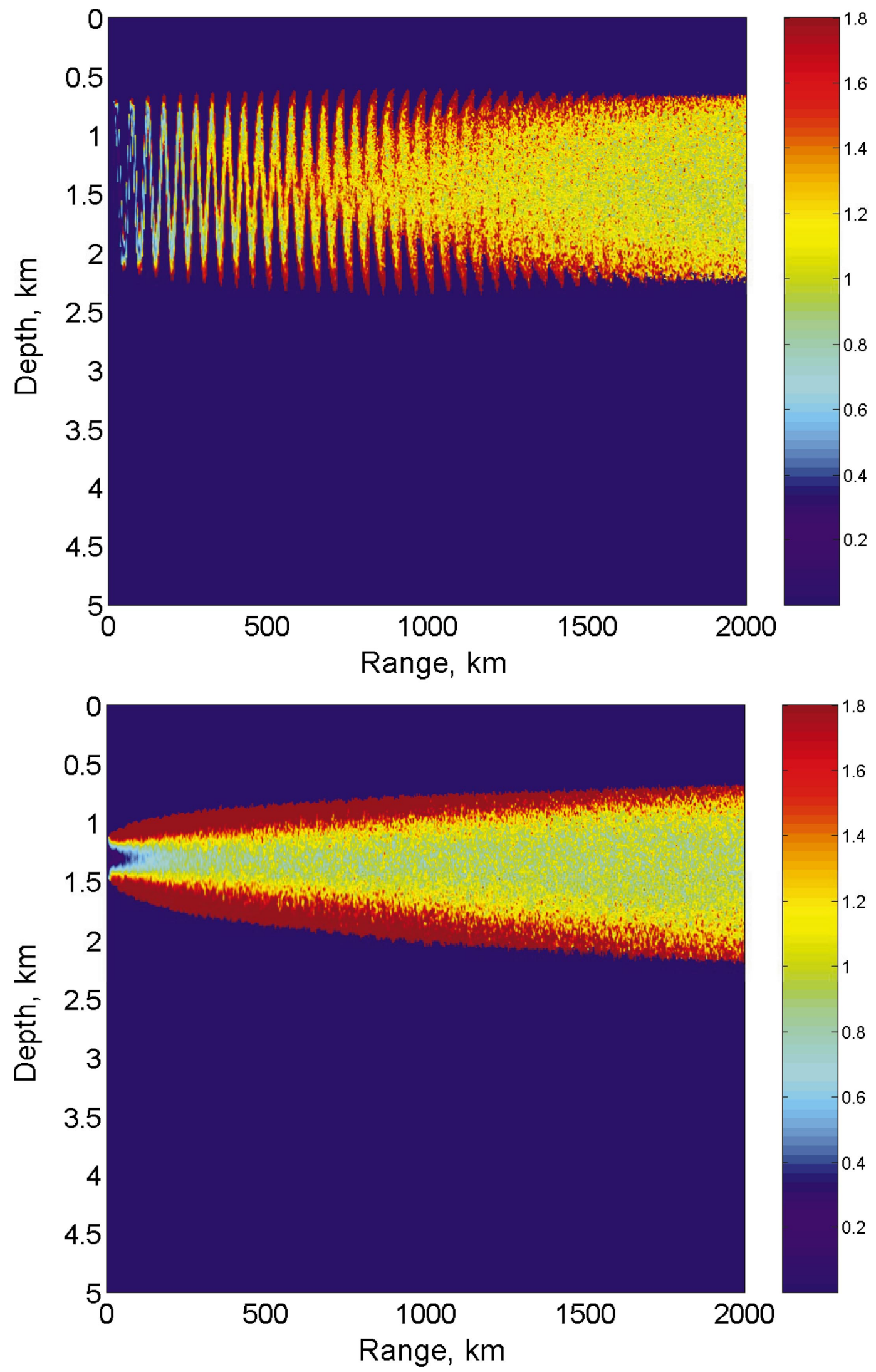

FIG. 6. Spatial distribution of scintillation index for the $250-\mathrm{Hz}$ beam with source depths of $1300 \mathrm{~m}$ (lower) and $2000 \mathrm{~m}$ (upper). Scintillation index is only computed where there is significant mean intensity (see Fig. 6); thus, regions of dark blue represent areas where the scintillation index is not well defined.

implying a diffusive process. The simulations at $125 \mathrm{~Hz}$, not shown here, have essentially identical beam-spreading curves, which suggests that the scattering is close to geometrical.

\section{WAVE FIELD COMPLEXITY AND ENTROPY}

A useful, though nonunique, representation of the simulated wave field complexity is sought. The complexity of a 


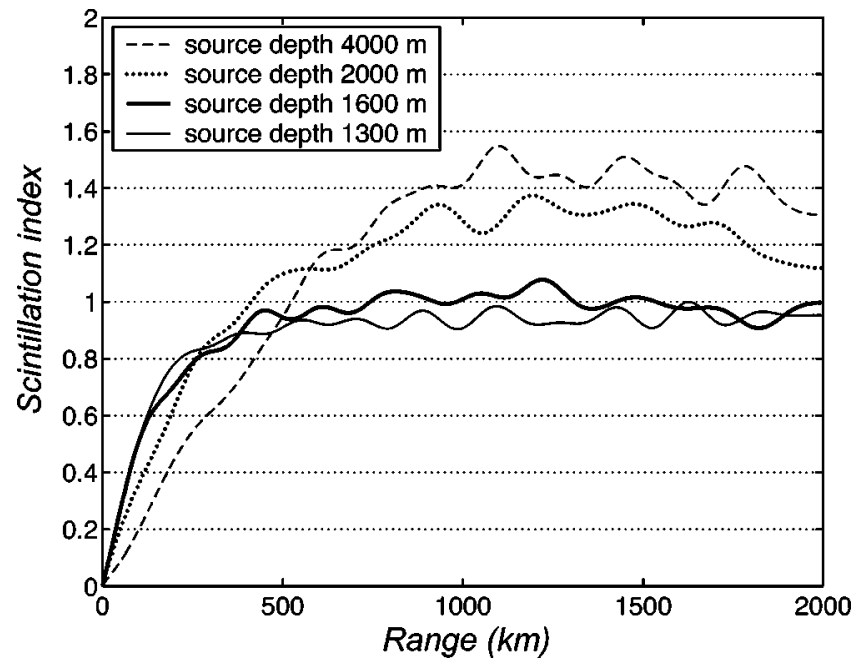

FIG. 7. Scintillation index of $250-\mathrm{Hz}$ beams for four different source depths, and computed only along a line defined by the centroid of the unperturbed narrow beams.

random process depends on the number of independent components, and one such representation has already been presented, namely the spatial spectrum of the beams, $S_{0}\left(r, k_{z}\right)$, where clearly a white spectrum has more complexity than a simple line spectrum. However, it proves useful to apply the Karhunen-Loeve (KL) expansion (Van-Trees, 1968), ${ }^{3}$ instead of the Fourier expansion because the KL expansion yields uncorrelated components in this nonstationary environment. The complex envelope of the sound-pressure field at each range step can be represented by a stochastic series with $M(r)$ significant contributions. $M(r)$ will be called the dimension of the random process, with

$$
\begin{aligned}
& \psi(r, z)=\sum_{j=1}^{M(r)} \alpha_{j}(r) \Phi_{j}(r, z) \\
& \alpha_{j}(r)=\int_{-H}^{0} \psi(r, z) \Phi_{j}(r, z) d z, \\
& \lambda_{j}(r) \Phi_{j}(r, z)=\int_{-H}^{0} K(z, \tilde{z}, r) \Phi_{j}(r, \tilde{z}) d \tilde{z}, \\
& K(z, \tilde{z}, r)=\left\langle\psi(r, z) \psi^{*}(r, \tilde{z})\right\rangle,
\end{aligned}
$$

where $K(z, \tilde{z}, r)$ is the transverse correlation function at range $r$, and $\lambda_{j}(r)$ and $\Phi_{j}(r, z)$ are the eigenvalues and eigenfunctions of the KL expansion at range $r$, respectively. The functions $\lambda_{j}(r)$ and $M(r)$ are shown in Figs. 10 and 11. Note that curves for the angle spectrum width and number of significant KL expansion components both can be used as an approximate measure of random process.

Precisely, for a stochastic variable $\Gamma$, a logarithmic measure of the complexity of that variable is the entropy $E$

$$
E=C-I \text {, }
$$

where $C$ is a constant and $I$ is the Shannon information (Shannon, 1948) measured in bits

$$
I=-\int P(\Gamma) \log _{2}[P(\Gamma)] d \Gamma .
$$

Here, $P(\Gamma)$ is the probability density function (PDF). In this analysis, the complexity of the complex envelope as a function of depth $z$ at a given range $r$ is of interest; therefore, the Shannon information at a range $r$ is written

$$
\begin{aligned}
I(r)= & -\int P\left(\psi\left(r, z_{1}\right), \psi\left(r, z_{2}\right), \ldots\right) \\
& \times \log _{2}\left[P\left(\psi\left(r, z_{1}\right), \psi\left(r, z_{2}\right), \ldots\right)\right] d \psi\left(r, z_{1}\right) d \psi\left(r, z_{2}\right) \ldots \\
= & -\int \log _{2}[P(\psi(r, z)] d P(\psi(r, z)),
\end{aligned}
$$

where $P(\psi(r, z))$ is the probability density function for obtaining the vertical profile of $\psi$ at range $r$. The calculation of sound-pressure entropy directly by Monte Carlo stochastic simulation needs a large number of realizations to define $P(\psi)$ and is therefore computationally intensive. A simplified approach based on the Gaussian approximation of the acoustical fluctuations will be used, that is, the entropy will be calculated as if a process probability density function (PDF) is spatially Gaussian. Figure 7 shows that this is a correct assumption for the ranges where SI is close to 1 , that is, $r>200 \mathrm{~km}$. Since there is a one-to-one mapping between the coefficients of the KL decomposition and the complex envelope $\psi$, the entropy can be calculated as an entropy of a random vector of these coefficients. Generally, the probability density of a vector $X=\left(x_{1}, x_{2}, \ldots, x_{M}\right)$ of $M$ Gaussian complex random values with nonsingular correlation matrix $K_{X}$ has the following form:

$$
P=\frac{1}{\pi^{M} \operatorname{det}\left(K_{X}\right)} \exp \left(-\left(X-m_{x}\right)^{T} K_{X}^{-1}\left(X-x_{m}\right)\right),
$$

where $K_{X}=\left\langle\left(X-m_{x}\right)\left(X-m_{x}\right)^{T}\right\rangle$ is the complex correlation matrix of the complex random vector $X, m_{x}=\langle X\rangle$, and the superscript ${ }^{T}$ means complex conjugate and matrix transpose. The entropy, $E$, of a Gaussian random vector can be calculated analytically, yielding (Van-Trees, 1968; Cover and Thomas, 1991)

$$
E(r)=C-I(r)=C+\log _{2}\left(\pi^{M} \operatorname{det}\left(K_{X}\right)\right)+M \log _{2}(e) .
$$

In the case of a random vector of uncorrelated components of a KL expansion, the entropy is simply the sum of the entropies of the individual KL modes, namely

$$
\begin{aligned}
E(r) & =E\left(\lambda_{1}(r), \lambda_{2}(r), \ldots, \lambda_{M(r)}(r)\right) \\
& =C+\sum_{j=1}^{M(r)} \log _{2}\left(e \pi \lambda_{j}(r)\right) \\
& =C+M(r) \log _{2}(e \pi)+\sum_{j=1}^{M(r)} \log _{2} \lambda_{j}(r) .
\end{aligned}
$$

For equipartition of energy, $\lambda_{j}=\lambda$ (eigenvalue spectrum "white") the entropy is maximum, but for any other eigenvalue spectrum the entropy is less than this maximum value. The eigenvalue spectrum from the $250-\mathrm{Hz}$ simulation with source depth at $2000 \mathrm{~m}$ is shown in Fig. 10. The spectrum expands with range, but even at the distance $2000 \mathrm{~km}$ it remains nonuniform ("not white"). Note that this simple 

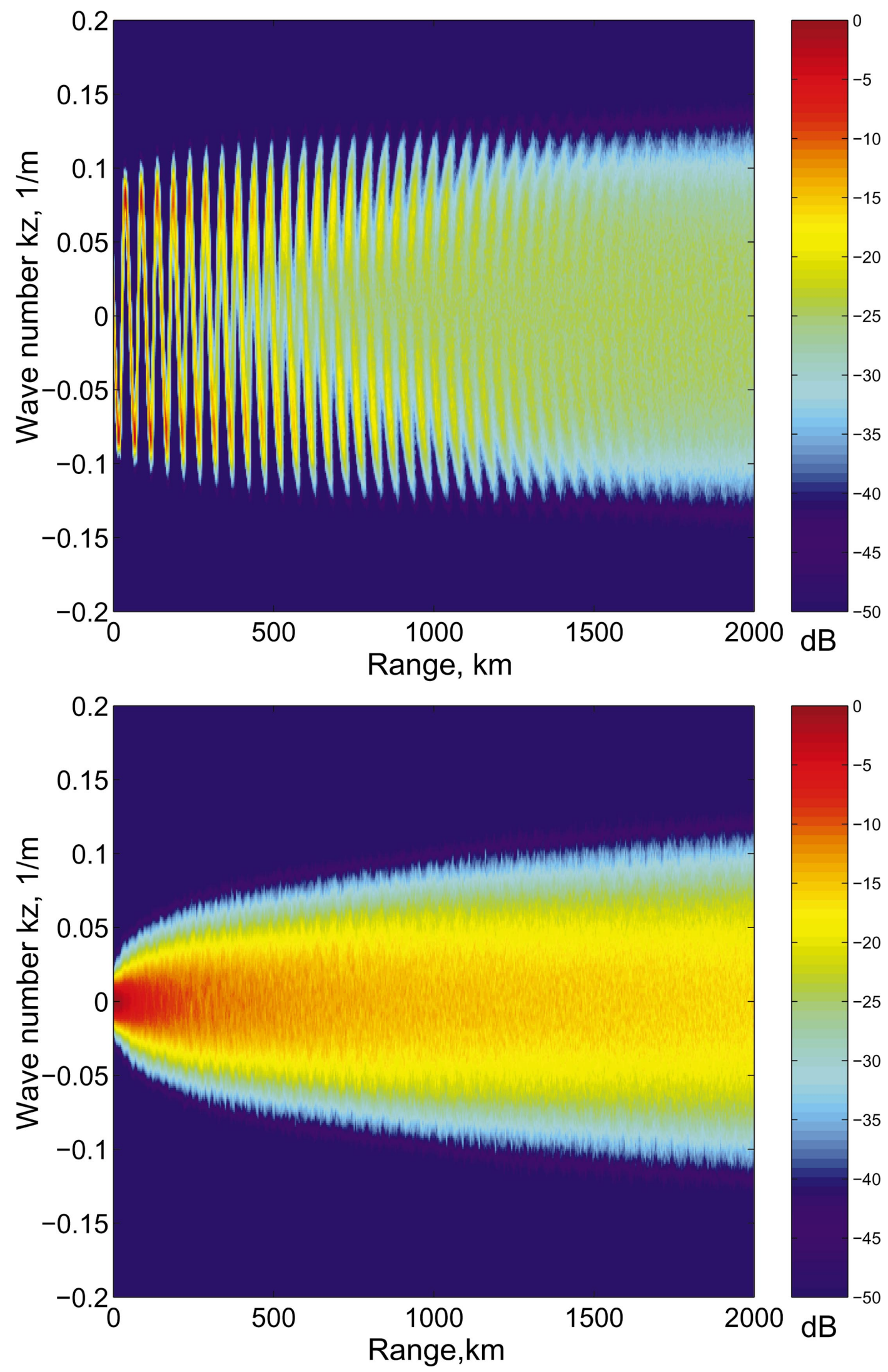

FIG. 8. Range evolution of the beam wave number $k_{z}$ spectrum of the $250-\mathrm{Hz}$ beam with source depths of $2000 \mathrm{~m}$ (upper) and $1300 \mathrm{~m}$ (lower).

definition of entropy is based on the assumption that the Gaussian random process is nonsingular; otherwise, a more rigorous definition must be applied in terms of absolute continuity of probability measures and Radon-Nikodim derivative (Halmos, 1950).
The entropy of the $250-\mathrm{Hz}$ weakly divergent sound beams is shown in Fig. 12 for beams with different sound source depths. In all cases $C=0$ and $M(r)=100$, which is larger than the maximum number of significant KL eigenvalues at any range and for any of the acoustical beams. The 

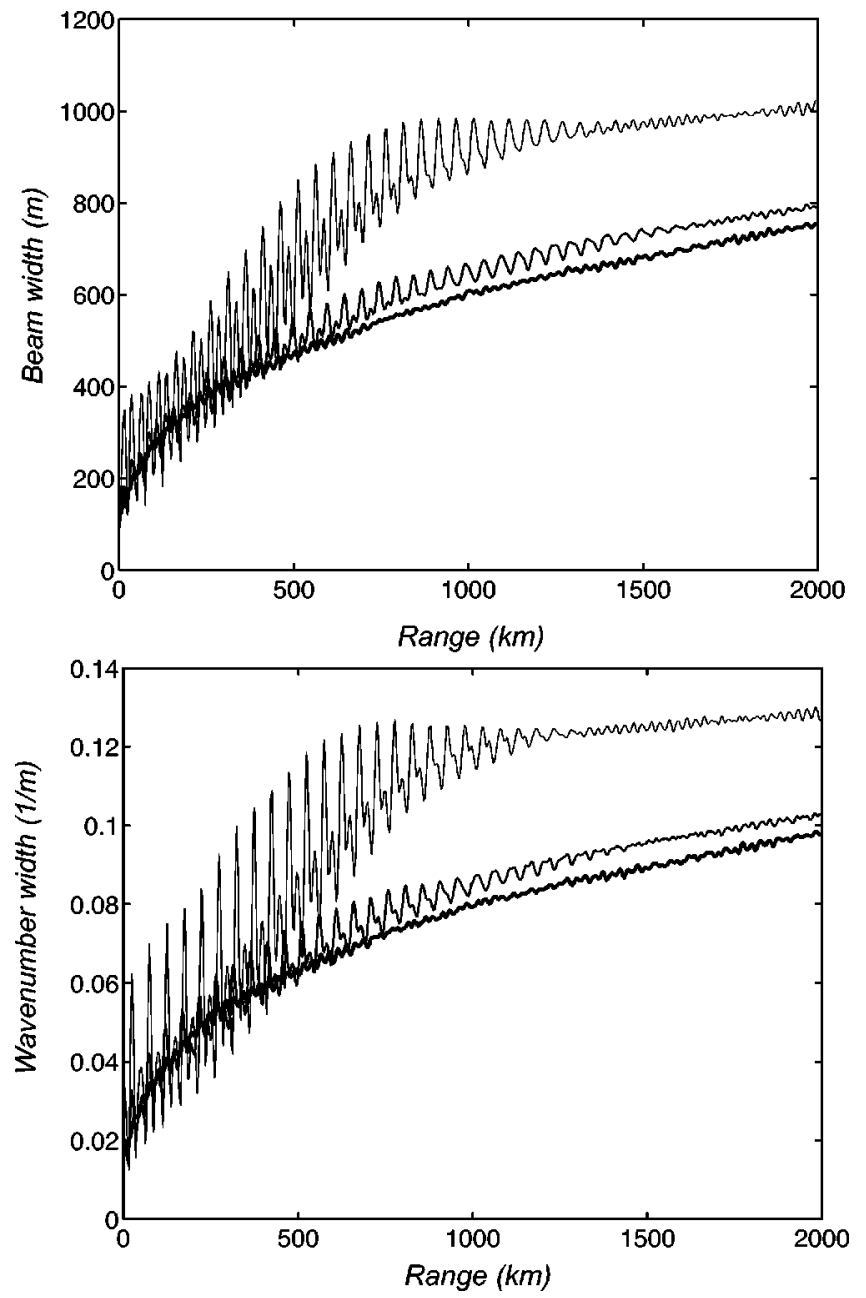

FIG. 9. Acoustical beamwidth, $w(r)$ (upper) and spectral beamwidth $w_{s}(r)$ (lower) as a function of propagation range for the $250-\mathrm{Hz}$ beams at source depths of $1300 \mathrm{~m}$ (thick line), $1600 \mathrm{~m}$ (medium line), and $2000 \mathrm{~m}$ (thin line).

entropy was calculated for the ranges where the unperturbed beam crosses the sound-channel axis (i.e., 1300-m depth). This approach eliminates the deterministic oscillations of entropy at the shorter ranges, an effect seen in Fig. 9. At ranges

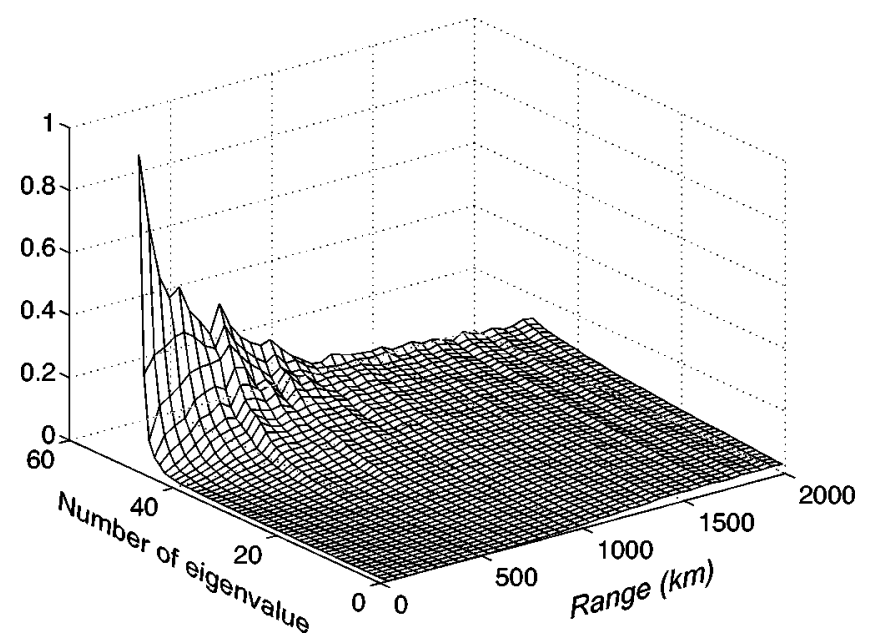

FIG. 10. Karhunen-Loeve (KL) eigenvalue spectrum as a function of range for the $250-\mathrm{Hz}$ beam with source depth at $2000 \mathrm{~m}$.

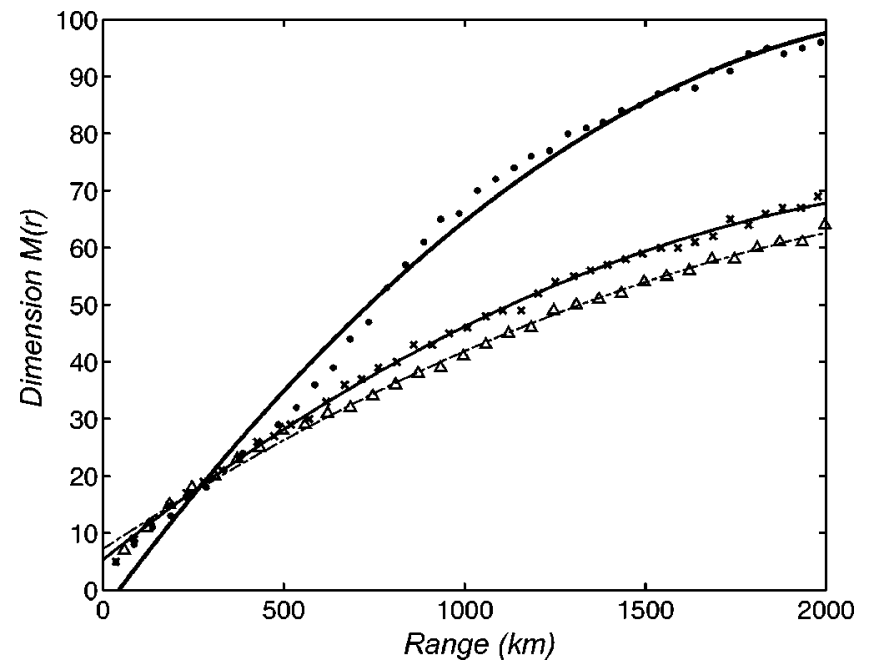

FIG. 11. Dimension $M(r)$ of random beams as a function of range. Beams at different source depths are $1300 \mathrm{~m}$ (thick line:axial), $1600 \mathrm{~m}$ (medium line), and $2000 \mathrm{~m}$ (thin line).

larger than roughly $1000 \mathrm{~km}$, the entropy is increasing monotonically and approximately linearly for all beams, and the entropy rates in the linear regime are $0.11,0.11$, and 0.06 $\mathrm{bit} / \mathrm{km}$ for the 1300-, 1600-, and 2000-m source depths, respectively. The scintillation index curves (Fig. 7) show that the linear behavior of entropy occurs very close to full saturation. Further, the linear rates are about the same for all beams, so that the anisotropy associated with beam angle is not strong, though primarily small-angle beams were considered. It must be noted that the initial rapid nonlinear rise of the entropy is an artifact of the Gaussian approximation in the entropy calculation; in the linear entropy region the Gaussian approximation is very good (see Fig. 7). Figure 12 also shows the entropy of a $125-\mathrm{Hz}$ beam with the sound source at $2000 \mathrm{~m}$, and there is virtually no difference be-

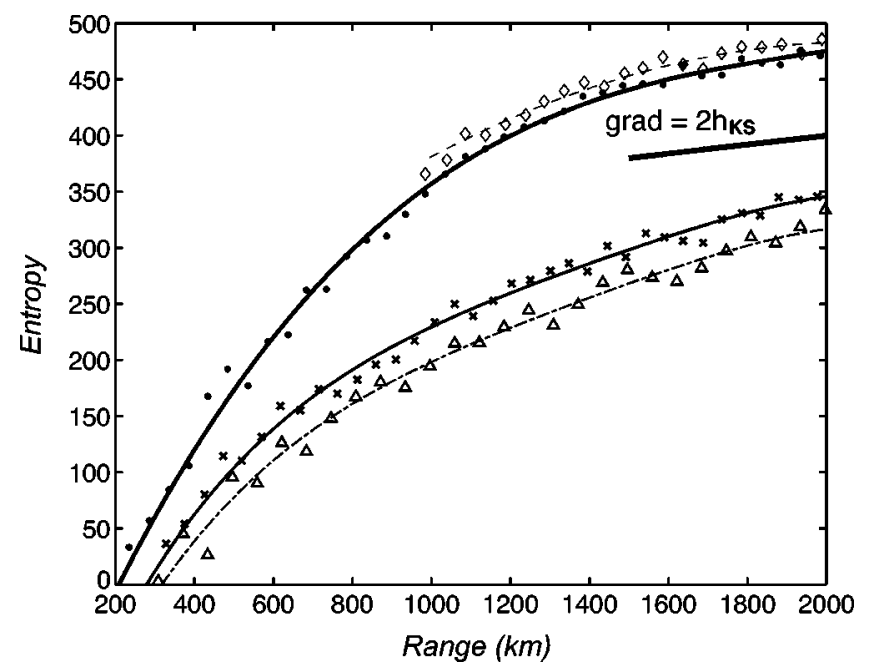

FIG. 12. Entropy of $250-\mathrm{Hz}$ acoustical beams for source depths of $1300 \mathrm{~m}$ (thick line circles), $1600 \mathrm{~m}$ (medium line crosses), and $2000 \mathrm{~m}$ (thin line triangles). Fits in the linear region of the curves yield entropy rates of 0.11 $(1300 \mathrm{~m}), 0.11(1600 \mathrm{~m})$, and $0.06(2000 \mathrm{~m}) \mathrm{bit} / \mathrm{km}$. The $2000-\mathrm{m}$ source depth, $125-\mathrm{Hz}$ entropy curve (arbitrarily vertical offset) is shown with a dash curve and diamond symbols. Also shown in solid is twice the KS entropy, $2 h_{\mathrm{KS}}=0.04 \mathrm{bit} / \mathrm{km}$ (arbitrary vertical offset) computed from the ray equations for the 1300-m axial ray. 
tween the long-range entropy rate at the two frequencies. The linear increase in $E(r)$ means that the number of equiprobable states of the dynamical system (complexity) is increasing exponentially and independent of acoustic frequency, in complete accordance with ray chaos predictions (Brown et al., 2003, Beron-Vera et al., 2003). The frequency independence of the entropy rate not only reinforces the geometric result but also suggests that these results carry over to broadband signals. In the next section it is determined if the rate of exponential increase in complexity computed from the full-wave simulations is comparable to the rate computed from ray theory.

\section{RAY ENTROPY}

It has been well established that ray trajectories in ocean acoustic propagation through internal waves are chaotic or unstable to perturbations in the medium and in the initial conditions (Brown et al., 2003; Beron-Vera et al., 2003). The chaotic nature of ray propagation in the ocean is quantified using the Lyapunov exponent $\nu_{L}$, which is derived from a stability analysis of the ray path (Brown et al., 2003), but it is often useful to express this instability using information theory (Latora and Baranger, 1999; Casati, 1996) such that the information $I(s)$ associated with a segment of ray trajectory of length $s$ is equal asymptotically to

$$
\lim _{|s| \rightarrow \infty} \frac{I(s)}{s}=h_{\mathrm{KS}},
$$

where $h_{\mathrm{KS}}$ is the Kolmogorov-Sinai (KS) entropy. Note here that KS entropy is actually an entropy rate. For bounded dynamical systems like underwater sound $\nu_{L} \simeq h_{\mathrm{KS}}$ (Gaspard, 1990). A consequence of the chaotic nature of ray paths is that the number of eigenrays connecting a source and a receiver will grow exponentially with range, leading to an exponential increase in wave field complexity. Thus, the exponential increase in wave field complexity is examined using the Lyapunov exponent (a measure of the KS entropy), which is to be compared to the computed rate of Shannon entropy. Another comparison between Shannon entropy and KS entropy for different dynamical systems was done by Latora and Baranger (1999).

The ray numerics are carried out as follows. Using the SPE Hamiltonian

$$
H(z, p ; r)=\frac{p^{2}}{2}+U(r, z),
$$

where $p=\tan \theta_{\text {ray }}$, the following well-known ray and stability equations are solved:

$$
\begin{aligned}
& \frac{d z}{d r}=\frac{\partial H}{\partial p}, \quad \frac{d p}{d r}=-\frac{\partial H}{\partial z}, \\
& \frac{d J}{d r}=K J,
\end{aligned}
$$

where

$$
J=\left(\begin{array}{ll}
\partial p /\left.\partial p_{0}\right|_{z_{0}} & \partial p /\left.\partial z_{0}\right|_{p_{0}} \\
\partial z /\left.\partial p_{0}\right|_{z_{0}} & \partial z /\left.\partial z_{0}\right|_{p_{0}}
\end{array}\right),
$$

$$
K=\left(\begin{array}{cc}
-\partial_{z p} H & -\partial_{z z} H \\
\partial_{p p} H & -\partial_{z p} H
\end{array}\right)=\left(\begin{array}{cc}
0 & -\partial_{z z} U \\
1 & 0
\end{array}\right) .
$$

The initial condition for $J$ at $r=0$ is the identity matrix. The Lyapunov exponent is derived from the stability equations, and is given by

$$
\nu_{L}=\lim _{r \rightarrow \infty} \frac{\log |\operatorname{Tr}(J)|}{r},
$$

where $\operatorname{Tr}(J)$ is the trace or sum over the diagonal elements of the stability matrix $J$ (Brown et al., 2003; Wolfson and Tomsovic, 2001). In the present calculation the asymptotic range result is not computed, however, and a finite range stability exponent defined by

$$
\nu=\frac{\log |\operatorname{Tr}(J)|}{r},
$$

is evaluated. This finite range stability exponent has the property that (Wolfson and Tomsovic, 2001)

$$
\nu_{L}=\langle\nu\rangle \sim h_{\mathrm{KS}},
$$

where the expectation values imply averaging over realizations of the ocean internal wave field. Last, the ray initial conditions are $z(0)=1300 \mathrm{~m}$ and $p(0)=0$, an axial ray.

An explicit adaptive Runge-Kutta algorithm was used to calculate 200 realization of ray trajectories with the above initial conditions. The second derivatives of $U(r, z)$ were calculated using cubic spline interpolation, and the base 2 logarithm was used in Eq. (35) to get result measured in $\mathrm{bit} / \mathrm{km}$ so as to be comparable with the Shannon entropy results. ${ }^{2}$ The calculation yields an estimate of $h_{\mathrm{KS}}$ of 0.020 $\mathrm{bit} / \mathrm{km}$ for the axial ray, decreasing linearly to $0.012 \mathrm{bit} / \mathrm{km}$ for the 2000-m source ray. These values are comparable to other calculations of Lyapunov exponent for ray propagation through ocean internal waves (Beron-Vera et al., 2003; Beron-Vera and Brown, 2003). In comparing this value to the computed gradients of Shannon entropy for the complex envelope, it must be noted that the complex envelope includes information about both phase and amplitude, while $h_{\mathrm{KS}}$ is only a measure of the sound-field energy characteristics, such as intensity and angular power density. So, the information gradient of the full-field entropy must be at least twice the KS entropy. Thus, $2 h_{\mathrm{KS}}=0.04 \mathrm{bit} / \mathrm{km}$ is placed in Fig. 12 for comparison with the full-wave Shannon entropy, and order of magnitude agreement with the axial beam is seen. It must be emphasized here that the comparison between KS entropy and Shannon entropy rate is crude as we have only compared the KS entropy of one ray, while the Shannon entropy involves a bundle of rays with a range (albeit small) of initial conditions. It is known, for example, that there can be significant variability of Lyapunov exponent as a function of initial conditions due to the structure of the background sound-speed profile (Beron-Vera et al., 2003; Beron-Vera and Brown, 2003).

The comparison of Shannon or physical entropies curves $E(r)$ of the complex envelope of the full-field simulation with $2 h_{\mathrm{KS}}$ shows that the evolution of narrow-beam entropy has two stages. In the first stage the beamwidth and spatial spectrum are growing rapidly with oscillations due to diffrac- 
tive effects. These effects are deemed diffractive because they are not described by the geometrical $h_{\mathrm{KS}}$ rate. The second stage occurs approximately near saturation; the entropy gradient slows down and tends to a level which is approximately twice the KS entropy of the corresponding chaotic ray trajectories. This second stage can be called chaotic from both a full-wave and ray perspective, because entropy is monotonically growing with the gradient determined by the average Lyapunov exponent. Simultaneously, beamwidth, spatial spectrum width, and dimension of the random process are all approximately linearly increasing. The stabilization of entropy gradient shows that, when fluctuations approach saturation, ray chaos phenomenon becomes the main factor determining the properties of scattering processes.

\section{SUMMARY AND DISCUSSION}

Monte Carlo stochastic simulation of acoustic scattering by internal waves allows analysis of the variability of weakly divergent beam as a function of range and beam angle. A realistic Garrett-Munk internal wave model for the induced sound-speed fluctuation was applied. The analysis was performed in terms of information theory using the physical or Shannon entropy of the complex acoustical field envelope as a logarithmic measure of wave chaos complexity and Kolmogorov-Sinai entropy as a logarithmic measure of ray chaos complexity. The relative intensity variance or scintillation index was used to quantify the approach to saturation, and for steep-angle beams SI approaches 1 from above, while for small-angle beams the approach is from below 1 . At the range near saturation the rate of Shannon entropy for the complex envelope of the finite-frequency acoustical beams decreases and converges to a constant value which is approximately twice as large as the Kolmogorov-Sinai entropy or average Lyapunov exponent associated with the central ray of that beam. This correspondence suggests a fullwave manifestation of ray chaos, but only after saturation of the scintillation index has occurred. In spite of this correspondence, the simulated acoustical beams are not seen to expand exponentially (or explosively), but they expand diffusively.

The results obtained in this analysis can be compared to the work of Wolfson and Tappert (2000), and Tappert (2003), who treat the problem of wave and ray chaos for constant background sound speed (no waveguide) and a ocean mesoscale-like single-scale random medium (Gaussian correlation function). Using the theory of Gaussian beams, they predict that the width of a narrow-angle beam will increase exponentially with range at a rate that is predicted by the geometric Lyapunov exponent, which they can compute analytically. The observation of this explosive growth of the beam is masked at short range by diffractive beam spreading, and at long range by a surmised breakdown of geometrical theory near saturation. The breakdown at long range has proved very difficult to estimate, while the short-range masking by diffraction is relatively straightforward.

In the present analysis, which involves the ocean waveguide and realistic internal-wave sound speed perturbations, a very different picture emerges. Because of the restoring force of the waveguide, a narrow-angle beam cannot spread exponentially, and thus the signature of chaos must be found in some other observable. It has been shown that such a signature is found in the wave field entropy. Like the nowaveguide case, at short ranges the signature of ray chaos is not manifest in the entropy due to deterministic and diffractive effects. However, once the wave field comes very close to saturation, the variation of entropy becomes linear with range roughly as predicted by the KS entropy. Thus, while the no-waveguide case suggests an arrest of ray chaos manifestations at saturation, the waveguide case examined here shows that near-saturation is an essential element. Clearly, the wave field entropy cannot grow indefinitely as this system is bounded by the ocean surface and bottom. We surmise that the entropy will grow linearly until acoustic energy fills the entire water column, at which time the entropy should stabilize.

It also should be emphasized that our results do not preclude the use of geometric methods for describing scattering phenomena at shorter ranges where the Shannon entropy rate and KS entropy do not agree. In fact, the $\sqrt{r}$ scaling of the beamwidth curves and their insensitivity to acoustic frequency (Fig. 9) suggest strongly that the scattering is geometric.

The numerical results of this paper require a theoretical foundation which we hope to formulate in future work.

\section{ACKNOWLEDGMENTS}

This work was inspired by Fred Tappert, and we have benefited from many useful discussions with Mike Wolfson. This is Woods Hole Oceanographic Institution contribution number 11208 .

${ }^{1}$ In ocean acoustic propagation the range variables, $r$, is equivalent to the time variable, $t$, in dynamical systems theory.

${ }^{2}$ The conversion from bits $/ \mathrm{km}$ to $\mathrm{km}^{-1}$ simply involves a change in the base of the logarithms. Thus, an entropy rate of 0.02 bits $/ \mathrm{km}$ would be $0.02\left(\log _{2} e\right)^{-1}=0.0139 \mathrm{~km}^{-1}$.

${ }^{3}$ In geophysics the Karhunen-Loeve (KL) expansion is often referred to as an empirical orthogonal function (EOF) expansion.

Beron-Vera, F. J., and Brown, M. G. (2003). "Ray stability in wekaly range dependent sound channels," J. Acoust. Soc. Am. 114(1), 123-130.

Beron-Vera, F. J., Brown, M. G., Colosi, J. A., Tomsovic, S., Virovlyansky, A., Wolfson, M. A., and Zaslavsky, G. M. (2003). "Ray dynamics in a long range acoustic propagation experiment," J. Acoust. Soc. Am. 114(3), 1226-1242.

Brekhovskih, L. M., Goncharov, V. V., and Kurtepov, V. M. (1995). "Weakly divergent bundles of sound rays in the Arctic," Atmos. Oceanic Phys. 31(3), 441-446.

Brown, M. G., Colosi, J. A., Tomsovic, S., Virovlyansky, A., Wolfson, M. A., and Zaslavsky, G. M. (2003). "Ray dynamics in a long range deep ocean sound propagation," J. Acoust. Soc. Am. 113(5), 2533-2547.

Casati, G. (1996). "Quantum chaos," Chaos 6(3), 391-398.

Casati, G., and Chirikov, B. V. (1995). Quantum Chaos: Between Order and Disorder: A Selection of Papers (Cambridge University Press, New York).

Colosi, J. A., and Brown, M. G. (1998). "Efficient numerical simulation of stochastic internal-wave induced sound-speed perturbation fields," J. Acoust. Soc. Am. 103(4), 2232-2235.

Colosi, J. A., and the ATOC Group (1999b). "A review of recent results on ocean acoustic wave propagation in random media: Basin scales," IEEE J. Ocean. Eng. 24(2), 138-155.

Colosi, J. A., Scheer, E. K., Flatte, S. M., Cornuelle, B. D., Dzieciuch, M. A., Munk, W. H., Worcester, P. F., Howe, B. M., Mercer, J. A., Spindel, R. C., Metzger, K., Birdsall, T. G., and Baggeroer, A. B. (1999a). "Compari- 
sons of measured and predicted acoustic fluctuations for a 3250-km propagation experiment in the eastern North Pacific Ocean," J. Acoust. Soc. Am. 105(6), 3202-3218.

Colosi, J. A., and Baggeroer, A. B. (2003). "On the kinematics of broadband multipath scintillation and the approach to saturation," J. Acoust. Soc. Am. 116(6), 3515-3522.

Cover, T. M., and Thomas, J. A. (1991). Elements of Information Theory (Wiley Science, New York).

Dzieciuch, M. A., Munk, W. H., and Worcester, P. F. (2001). "Turning point filters: Analysis of sound propagation on a gyre-scale," J. Acoust. Soc. Am. 110(1), 135-149.

Flatté, S. M., Dashen, R., Munk, W., Watson, K., and Zachariasen, F. (1979). Sound Transmission through a Fluctuating Ocean (Cambridge University Press, Cambridge).

Gaspard, P., and Nicolis, G. (1990). "Transport properties, Lyapunov exponents, and entropy per unit time," Phys. Rev. Lett. 65, 1693-1696.

Giannoni, M. J., Voros, A., and Zinn-Justin, J. (1989). Chaos and Quantum Physics (Elsevier Science, Amsterdam).

Goncharov, V. V., and Kurtepov, V. M. (1994). "Formation and propagation of weakly diverging bundles of rays in a horizontally inhomogeneous ocean," Acoust. Phys. 40(5), 685-692.

Halmos, P. R. (1950). Measure Theory (Springer-Verlag, New York).
Jensen, F. B., Kuperman, W. A., Porter, M. B., and Schmidt, H. (2000). Computation Ocean Acoustics, AIP Series in Modern Acoustics and Signal Processing (Springer, New York).

Latora, V., and Baranger, M. (1999). "Kolmogorov-Sinai entropy rate versus physical entropy," Phys. Rev. Lett. 82(3), 520-523.

Munk, W. H. (1976). "Sound channel in an exponentially stratified ocean, with application to SOFAR," J. Acoust. Soc. Am. 55(2), 220-226.

Petukhov, Y. V. (1994). "A sound beam with minimal wave front divergence in a stratified ocean waveguide," Acoust. Phys. 40(1), 97-105.

Shannon, C. E. (1948). "A mathematical theory of communication," Bell Syst. Tech. J. 27, 379-423.

Tappert, F. (2003). "Theory of explosive beam spreading due to ray chaos," J. Acoust. Soc. Am. 114(5), 2775-2781.

Topinka, M. A., and Westervelt, R. M. (2003). "Imaging electron flow," Phys. Today 56(12), 47-52.

Van-Trees, H. (1968). Detection, Estimation, and Modulation Theory (Wiley, New York).

Wolfson, M. A., and Tappert, F. D. (2000). "Study of horizontal multipaths and ray chaos due to ocean mesoscale structure," J. Acoust. Soc. Am. 107, $154-162$.

Wolfson, M. A., and Tomsovic, S. (2001). "On the stability of long-range sound propagation through a structured ocean," J. Acoust. Soc. Am. 109, 2693-2703. 